\title{
Editorial
}

\section{A general theory of smoothing and anti-smoothing, converting true returns to reported returns}

\author{
Journal of Derivatives \& Hedge Funds (2012) 18, 111-112. doi:10.1057/jdhf.2012.5;
}

published online 8 March 2012

Smoothing is a ubiquitous feature of financial return data, especially in the area of hedge funds and alternative assets, an area which is the remit of JDHF. There are separate literatures on the smoothing of real estate returns, hedge funds, mutual funds, private equity (PE), venture capital (VC), and no doubt, many other asset classes. The common feature is that the data collection is usually voluntary and self-reporting is involved.

Most existing smoothing models are implicitly linear. They propose an equation relating reported returns to true returns. The nature of this equation can usually be understood as a simple auto-regressive moving average model, where the true return is the error term and the reported return is the observed variable. Wellknown examples in the literature are real estate where the standard practice is to use an AR (1) and hedge funds where an MA (n) is employed.

I view smoothing as a mechanism that converts true returns into reported returns. The two may differ because of appraisal, stale data, misreporting and conservatism in marking to market; indeed, there may be other explanations and the ones listed by me may not be mutually exclusive. These causes will vary from asset class to asset class; for example, while appraisal seems a key feature of PE data, it does not appear relevant to VC.The true returns are often unobservable and often endowed with certain properties according to the philosophical beliefs of the scholar. A common assumption is that one may wish to assume that true returns are white noise because of the operation of efficient markets.

Not all smoothing mechanisms for financial returns are linear. Regime switching is a useful mechanism for capturing different market conditions. One of the features of regime-switching models is that their steady-state distributions are typically multimodal. In fact, one can use this information informally as a preliminary investigative tool. VC seems a good candidate for multiple regime modelling, as Woodward and Hall (2003) note:

The data do not pass any test of lognormality. To the naked eye, not only are the distributions fat-tailed, they are mildly bi-modal. To assure that this phenomenon was not coming from dirt in the data (such as typographical errors) we undertook a special review of the 100 companies who contribute the most value (about 35 percent) to the index. All were confirmed to be real events (Woodward and Hall, 2003, p. 18)

This topic is under-researched, here are a number of issues worth investigating; under what conditions are reported returns more volatile than true returns? Under what conditions are reported returns over and under-reporting true 
returns? The literature is not entirely clear as to when smoothing/conversion will reduce volatility. As smoothed returns enter into optimisation/asset allocation calculations, these issues have practical importance.

\section{REFERENCE}

Woodward, S.E. and Hall, R.E. (2003) Benchmarking the Returns to Venture. Sand Hill Discussion Paper.

Stephen Satchell 